\title{
The effect of breed and parity on curves of body condition during lactation estimated using a non-linear function
}

\author{
N.C. Friggens ${ }^{\dagger}$ and J.H. Badsberg \\ University of Aarhus, Faculty of Agricultural Science, Research Centre Foulum, PO Box 50, DK - 8830 Tjele, Denmark
}

(Received 29 August 2006; Accepted 16 January 2007)

The objectives of this study were to see if the body condition score curve during lactation could be described using a model amenable to biological interpretation, a non-linear function assuming exponential rates of change in body condition with time, and to quantify the effect of breed and parity on curves of body condition during lactation. Three breeds were represented: Danish Holstein $(\mathrm{n}=112)$, Danish Red $(\mathrm{n}=97)$ and Jerseys $(\mathrm{n}=8)$. Cows entered the experiment at the start of first lactation and were studied during consecutive lactations (average number of lactations 2, minimum 1, maximum 3). They remained on the same dietary treatment throughout. Body condition was scored to the nearest half unit on the Danish scale (see Kristensen (1986); derived from the Lowman et al. (1976) system) from 1 to 5 on days: 2, 14, 28, 42, 56, 84, 112, 168, 224 after calving. Additionally, condition score was recorded on the day of drying off the cow, 35, 21, and 7 days before expected calving and finally on the day of calving. All condition scores were made by the trained personal on the research farm, where the same person made $92 \%$ of the scores. The temporal patterns in condition score were modelled as consisting of two underlying processes, one related to days from calving, referred to as lactation only, the other to days from (subsequent) conception, referred to as pregnancy. Both processes were assumed to be exponential functions of time. Each process was modelled separately using exponential functions, i.e. one model for lactation only and one for pregnancy, and then a combined model for both lactation only and pregnancy was fitted. The data set contained 467 lactation periods and 378 pregnancy periods. The temporal patterns in condition score of cows kept under stable and sufficient nutritional conditions were successfully described using a two component non-linear function. First lactation cows had shallower curves, they had greater condition scores at the nadir of the curve. Danish Holstein and Jersey were thinner at the end of the mobilisation period having lost more body condition than the Danish Red breed. Although the dairy breeds ended up being thinner there were no significant differences in the rate at which they lost body condition.

Keywords: body condition, breed, dairy cows, non-linear model, parity

\section{Introduction}

Dairy cows generally respond to a poor nutritional environment by increasing mobilisation of body energy reserves. However, excessive mobilisation of body reserves in early lactation has been associated with increased health problems and a reduction in reproductive performance (Correa et al., 1990; Jorritsma et al., 2001). Early identification of cows that are likely to have an excessive body energy mobilisation would be a valuable management tool.

In order to quantify 'excessive' mobilisation it is first necessary to know what is normal body energy mobilisation. There is a characteristic pattern of change in body fatness through the reproductive cycle of pregnancy and

\footnotetext{
${ }^{\dagger}$ E-mail: N.Friggens@agrsci.dk
}

lactation. During pregnancy there is an increase in body fatness and during the first part of lactation there is a decrease in body fatness (Friggens et al., 2004). Until recently it was assumed that this mobilisation of body energy reserves is entirely a response to a shortfall in food energy intake relative to milk energy output. However, this assumption has been increasingly questioned. There are endocrine changes in pregnancy and lactation that facilitate changes in body lipid and there are sound evolutionary arguments for the strategic use of body reserves in support of lactation (see Chilliard et al. (2000); Friggens et al. (2004)). Significant genetic correlations between condition score measurements through lactation have also been reported (Coffey et al., 2001; Pryce et al., 2002). This evidence strongly suggests that a significant component of the observed pattern of change in body 
fatness through the reproductive cycle is a characteristic of the animal, i.e. it is genetically driven. Given this, it may be expected that significant differences exist in these genetically driven condition score curves between breeds and parities. Thus, the main objective of this study was to quantify the effects of breed and parity on curves of body condition during lactation.

Achieving this objective in such a way as to allow biological insight raises some challenges for the analysis of the data. Longitudinal data such as a time-series of condition scores can be analysed relatively easily by fitting linear functions, usually polynomials, using for example random regression models (e.g. Banos et al., 2005). However, such linear models have a number of limitations, particular in relation to their biological interpretation. It has been demonstrated, for lactation curves of milk yield, that there can be substantial benefits of using models that a biological basis, even if this means using non-linear models (Dijkstra et al., 1997; Hansen et al., 2006).

In the case of changing body fatness, it has been argued that the lactation curve is a composite of two processes, a non-linear decay in body fatness relative to days from calving followed by a non-linear increase in body fatness relative to days from the subsequent conception (Friggens et al., 2004). Accordingly, there is value in describing curves of body condition during lactation in terms that are consistent with existing theoretical descriptions as this facilitates the use of this information in a wider context. For example, with very few exceptions (e.g. Petruzzi et al., 2004), current methods to predict energy requirements are based solely on estimates of milk production, milk composition and maintenance. They do not explicitly allow for any genetically driven body energy mobilisation. Whilst it has been shown that prediction of the cow's energy requirements can be substantially improved, particularly in early lactation, by incorporating genetically driven body energy mobilisation (Friggens et al., 2004), it is not clear how to incorporate this effect in such a way as to allow modification of the curves for factors such as breed and parity. Although the differences due to breed and parity in body condition score curves have been described using linear models (e.g. Mao et al., 2004) it is not easy to deduce which underlying properties of the curves are affected. Fitting a non-linear model may provide a simple means to do this. Thus, the secondary objective of this study was to see if the curves of body condition during lactation could be described using a model amenable to biological interpretation, a non-linear function assuming exponential rates of change in body condition with time.

\section{Material and methods}

The data used in this study were collected within a 5-year experiment carried out from October 1996 to October 2001 at the Danish Cattle Breeders Organisation research farm, Ammitsbøl Skovgård. All the procedures involving animals were approved by the Danish Animal Experiments Inspectorate and complied with the Danish Ministry of Justice law no. 382 (10 June, 1987) and Acts 739, (6 December, 1988) and 333 (19 May, 1990) concerning animal experimentation and care of experimental animals.

\section{Experimental design and animals}

The design and methods for the production aspects of the experiment have been described in detail elsewhere (Nielsen et al., 2003). Briefly, three breeds were represented: Danish Holstein $(n=112)$, Danish Red $(n=97)$ and Jerseys $(n=78)$. A summary of the performance of the different breeds is presented in Table 1. Within breeds, equal numbers of cows were assigned to one of two dietary treatments. Cows entered the experiment at the start of first lactation and were studied during consecutive lactations (average number of lactations 2, minimum 1, maximum 3). They remained on the same dietary treatment throughout. The cows were housed throughout the year in single tie stalls.

\section{Feeding}

The cows were fed ad libitum one of two feeds, a normal energy density total mixed ration and a lower energy density total mixed ration. In the dry period ( 56 days prior to calving) all cows were fed the lower energy density ration. The two rations used the same concentrate and had the same forage:concentrate ratio. The forages used were ( $\mathrm{kg} / \mathrm{kg}$ dry ration): whole-crop pea silage (0.08 or 0.10$)$, whole-crop wheat silage $(0.305$ or 0.415$)$ and chopped straw ( 0.13 or 0$)$ in the lower and normal energy density rations, respectively. The concentrate composition was (kg/kg dry ration): rapeseed meal, 0.13 ; soya-bean meal, 0.05 ; sugar-beet pulp, 0.16 ; sugar-beet molasses, 0.125 ; mineral-vitamin mix, 0.02. The composition of the two rations was fixed irrespective of stage of lactation. The average digestible energy contents of the normal energy density ration and the lower energy density ration were 13.55 and $12.88 \mathrm{MJ} / \mathrm{kg}$ dry matter (DM), respectively. The average crude protein contents of the normal energy density ration and the lower energy density ration were 153 and $145 \mathrm{~g} / \mathrm{kg} \mathrm{DM}$, respectively.

Table 1 A summary of the average performance of the different breeds on the normal energy density feed in the present experiment (for further details see Nielsen et al., 2003)

\begin{tabular}{lccc}
\hline \hline & \multicolumn{3}{c}{ Breed } \\
\cline { 2 - 4 } & Danish Red & Danish Holstein & Jersey \\
\hline 305-day milk yield $(\mathrm{kg})^{\dagger}$ & 6060 & 7242 & 5081 \\
Milk fat $(\mathrm{g} / \mathrm{kg})$ & 47.0 & 45.2 & 61.8 \\
Milk protein $(\mathrm{g} / \mathrm{kg})$ & 37.0 & 35.4 & 41.7 \\
DM Intake $(\mathrm{kg} /$ day) & 18.8 & 20.8 & 16.4 \\
Liveweight $(\mathrm{kg})$ & 630 & 616 & 424 \\
\hline \hline
\end{tabular}

${ }^{\dagger}$ Excludes cows with records missing before 30 days in lactation. 
Body condition score measurements

Body condition was scored to the nearest half unit on the Danish scale (see Kristensen (1986); derived from the Lowman et al. (1976) system) from 1 to 5 on days: 2, 14, 28, $42,56,84,112,168,224$ after calving. Additionally, condition score was recorded on the day of drying off the cow, 35,21 , and 7 days before expected calving and finally on the day of calving. All condition scores were made by the trained personal on the research farm, where the same person made $92 \%$ of the scores. The condition scorer was calibrated against an external assessor at intervals of approximately 1 year. The external assessor scored on a wide range of farms and was involved in training of new condition scorers.

\section{Statistical analysis}

The temporal patterns in condition score were modelled as consisting of two underlying processes, one related to days from calving, referred to as lactation only, the other to days from (subsequent) conception, referred to as pregnancy. Both processes were assumed to be exponential functions of time. Each process was modelled separately, i.e. one model for lactation only and one for pregnancy, and then a combined model for both lactation only and pregnancy was fitted. In the combined model, the two time scales; days from calving and days from conception were retained. Only observations from the first 180 days in the lactation and before 4 weeks into the pregnancy were used for the lactation only period. Observations from 4 weeks before the pregnancy and until day 300 of lactation were used for the pregnancy period. All observations were used when the data from these two periods were combined in one model. The data set contained 467 lactation periods and 378 pregnancy periods.

Let $y_{i j}$ denote the jth measurement of the response, that is the body condition score, at time $t_{i j}, j=1, \ldots, n_{i}$ in the individual cow-lactation $i$. For each individual cow-lactation (or pregnancy in the pregnancy model), the parity (i.e. the reproductive cycle number) $p$, and the breed $b$, were known. These were used to estimate the components of the model parameters described below using non-linear models for repeated measurements (see Davidian and Giltinan, 2003) as implemented by PROC NLMIXED in SAS (Littell et al., 2006).

Model from calving to conception - lactation only From the calving to the pregnancy we assumed

$$
y_{i j}=\exp \left(A_{i}\right)+\exp \left(R_{i}\right)^{*} \exp \left(-\exp \left(l_{i}\right)^{*} t_{i j}\right)+\varepsilon_{i j}, j=1, \ldots, m_{i}
$$

with $\varepsilon_{i j} \approx N\left(0, \sigma_{\varepsilon}\right)$ and independent, and the triplet $\left(A_{i r} R_{i i}\right.$ $\left.l_{i}\right)$ depending on the individual lactation.

This is the simple exponential form $\left(Y=a+b \cdot e^{(-1 \cdot t)}\right)$ but with the coefficients expressed as exponents. The exponential form was chosen because it is simple (few coefficients) and has parameters that estimate key components of biological descriptions of body fat change (e.g. Friggens et al., 2004). The parameters of the curve of condition score during lactation are shown in Figure 1. The quantity $\exp \left(A_{i}\right)$ is the asymptote, the level achieved after a long time. The difference between level at (preceding) calving and asymptote is denoted by $\exp \left(R_{i}\right)$, with $\exp \left(A_{i}\right)+\exp \left(R_{i}\right)$ the level at the calving. Finally, the rate of decline is determined by $\exp \left(I_{i}\right)$. We modelled the curve using three exponential parameters to ensure that the coefficients were always positive, that is, to ensure the right shape of the curve. (We might thus also expect better normality of the random effects.) Optimisation of the model was done using the logarithm of the three exponential parameters.

The components of each parameter were modelled as:

$$
\begin{aligned}
A_{i} & =A+A_{b}+A_{p}+A_{b p}+\alpha_{i}, \\
R_{i} & =R+R_{b}+R_{p}+R_{b p}+\beta_{i}, \text { and } \\
I_{i} & =I+I_{b}+I_{p}+I_{b p}+\lambda_{i}
\end{aligned}
$$

where $A$ is the mean asymptote, $A_{b}$ the effect of breed on asymptote, $A_{p}$ the effect of parity on asymptote, $A_{b p}$ the effect of the interaction of breed and parity on asymptote, and $\alpha_{i}$ the random effect on the asymptote of the individual cow $\times$ lactation $i$. Similarly for $R_{i}$ and $l_{i}$.

We assumed the three random effects $\left(\alpha_{i}, \beta_{i}, \lambda_{i}\right)$ are multivariate normal:

$$
\left(\alpha_{i}, \beta_{i}, \lambda_{i}\right) \approx N_{3}(0, \Sigma) \text { with } \Sigma=\left|\begin{array}{ccc}
\sigma_{\alpha}^{2} & \rho_{\alpha \beta} & \rho_{\alpha \lambda} \\
\rho_{\alpha \beta} & \sigma_{\beta}^{2} & \rho_{\beta \lambda} \\
\rho_{\alpha \lambda} & \rho_{\beta \lambda} & \sigma_{\lambda}^{2}
\end{array}\right| .
$$

Model from conception to calving - pregnancy

We assumed:

$$
\begin{aligned}
y_{i j}= & \exp \left(A_{i}\right)+\exp \left(S_{i}\right) * \exp \left(-\exp \left(k_{i}\right) *\left(283-s_{i j}\right)\right) \\
& +\varepsilon_{i j}, j=m_{i}+1, \ldots, n_{i},
\end{aligned}
$$

with $\varepsilon_{i j} \approx N\left(0, \sigma_{\varepsilon}^{2}\right)$ and independent, and the triplet $\left(A_{i r} S_{i r}\right.$ $k_{i}$ ) depending on the individual cow-lactation.

With $s_{i j}=t_{i j}-t_{\text {conceive(i) }}$ the number of days into the pregnancy, $-\exp \left(k_{i}\right) *\left(283-s_{i j}\right)$ becomes $\exp \left(k_{i}\right)^{*}\left(t_{i j}-(283+\right.$ $\left.\left.t_{\text {conceive(i) }}\right)\right)$, and we thus have an increasing function with rate determined by $k_{i r}$ an asymptote of $\exp \left(A_{i}\right)$, and the level $\exp \left(A_{i}\right)+\exp \left(S_{i}\right)$ at expected calving, i.e. 283 days from conception (see Figure 1).

The triplet $\left(A_{i} S_{i}, K_{i}\right)$ was assumed to depend on breed and parity as above:

$$
\begin{aligned}
A_{i} & =A+A_{b}+A_{p}+A_{b p}+\alpha_{i r} \\
S_{i} & =S+S_{b}+S_{p}+S_{b p}+\xi_{i i} \text { and } \\
k_{i} & =k+k_{b}+k_{p}+k_{b p}+\kappa_{i r}
\end{aligned}
$$


Friggens and Badsberg

A

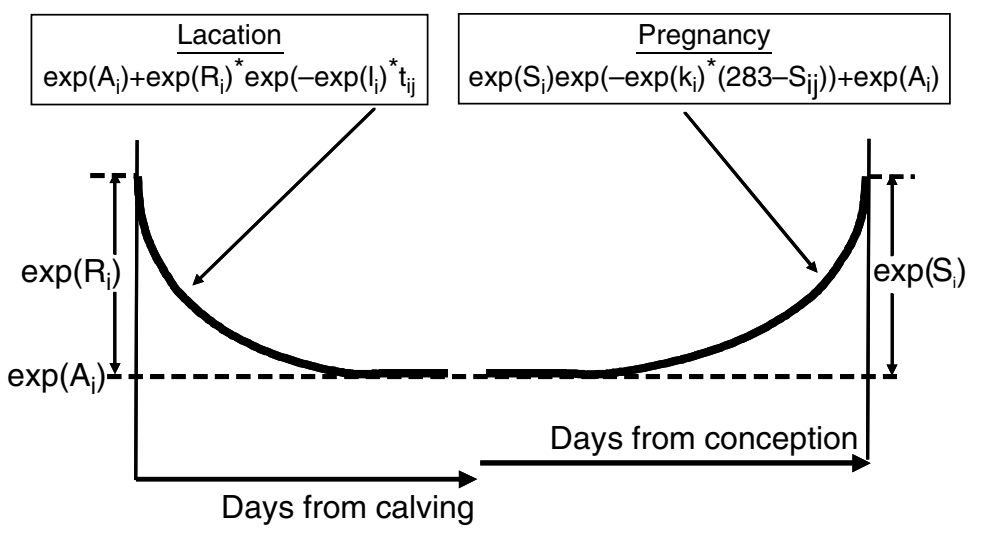

B

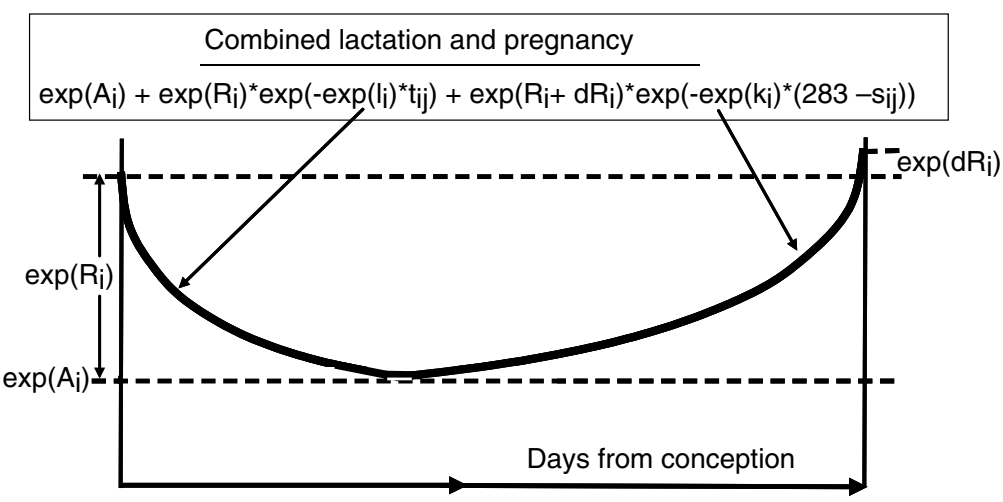

Days from calving

Figure 1 Schematic representations of the exponential equations describing condition score patterns for (A) the lactation only and pregnancy models, and (B) the combined model. Equation notation is as detailed in Material and methods (days from calving is denoted by $t$, days from conception is denoted by $s$ ).

and we assumed the three random effects $\left(\alpha_{i r} \xi_{i r} \kappa_{i}\right)$ are multivariate normal:

$$
\left(\alpha_{i}, \xi_{i}, \kappa_{i}\right) \approx N_{3}(0, \Sigma) \text { with } \Sigma=\left|\begin{array}{ccc}
\sigma_{\alpha}^{2} & \rho_{\alpha \xi} & \rho_{\alpha \kappa} \\
\rho_{\alpha \xi} & \sigma_{\xi}^{2} & \rho_{\xi \kappa} \\
\rho_{\alpha \kappa} & \rho_{\xi \kappa} & \sigma_{\kappa}^{2}
\end{array}\right| .
$$

Combined model, from calving to next calving

We assumed:

$$
\begin{aligned}
y_{i j}= & \exp \left(A_{i}\right)+\exp \left(R_{i}\right) * \exp \left(-\exp \left(l_{i}\right) * t_{i j}\right) \\
& +\exp \left(R_{i}+\partial R_{i}\right) * \exp \left(-\exp \left(k_{i}\right) *\left(283-s_{i j}\right)+\varepsilon_{i j},\right.
\end{aligned}
$$

for $j=1, \ldots m_{i}, m_{i}+1, \ldots, n_{i}$ with $\varepsilon_{i j} \approx N\left(0, \sigma_{\varepsilon}^{2}\right)$ and independent and the five parameters $\left(A_{i}, R_{i}, \partial R_{i}, l_{i}, k_{i}\right)$ depending on the individual pregnancy (lactation). $s_{i j}$ is as above, and $S_{i}$ from the pregnancy model is here given as $R+\partial R_{i}$ to get independent estimates.

Again $\left(A_{i}, R_{i}, \partial R_{i}, l_{i} k_{i}\right)$ are assumed to depend on breed and parity as above, and we assume the five random effects $\left(\alpha_{i}, \beta_{i}, \xi_{i}, \lambda_{i}, \kappa_{i}\right)$ are multivariate normal: $\left(\alpha_{i r}, \beta_{i,} \xi_{i}, \lambda_{i,} \kappa_{i}\right) \approx N(0, \Sigma)$.

By setting the first derivative of the above expression for the combined curve to zero we find minimum body condition score at time from:

$$
T_{\min }=\frac{R_{i}-\left(R_{i}+\partial R_{i}\right)+l_{i}-k_{i}+\exp \left(k_{i}\right) *\left(283+t_{\text {concieve }(i)}\right)}{\exp \left(l_{i}\right)+\exp \left(k_{i}\right)} .
$$

Method for fitting the models

According to Wolfinger (1999) 'PROC NLMIXED is best suited for models with a single random effect, although you can also successfully compute integrals in two and three dimensions as well.... Problems which are badly scaled or sufficiently noisy will not perform well with PROC NLMIXED.' Given that we were looking at three and fivedimensional problems, and that body condition score is relatively noisy, model fitting was not straightforward.

By finding very good starting values and by using the first order method for approximation of the likelihood we succeeded in fitting the models. To find starting values, we first fitted individual models to the individual lactation, one by one. The average parameters found for the fitted lactations were used for those lactations for which no fit was found. By iterating this procedure, progressively more and more models were fitted. The distribution of these parameters was then used for starting values in models 
with random effects. Here we first fitted the smaller models (lactation only and pregnancy), and used the parameters found for these models as starting values in the larger combined models, with the additional parameters set to a starting value of zero.

Akaike's information criterion (AIC) was used to evaluate model fit and thus to select between models. AIC represents a trade-off between how well the model fits to data (in terms of the magnitude of the log likelihood function) and the complexity of the model (in terms of the number of parameters). Contrary to most statistical model testing procedures (e.g. F tests, etc.), AIC does not depend upon that the models to be compared are nested and does not suffer from the classical problems concerning multiple testing (Burnham and Anderson, 2002). Given this, we chose to use AIC for evaluating the goodness of fit rather than other statistical tests that tend to inflate the significance of model additions. In all the models we evaluated, the AIC for a null model, i.e. containing no fixed effects, is given. Within the same data set, differences in AIC between two models that are greater than three indicate that there is good evidence that the model with the smaller AIC is significantly better than the model with the larger AIC (Burnham and Anderson, 2002).

\section{Results}

As the process of fitting non-linear models with random effects is relatively new within agricultural science (e.g. Bermejo et al., 2003), results regarding the model fitting are presented as well as the biological aspects.

\section{Model fitting}

The fitting was carried out on three data sets: the lactation only period before pregnancy using days from calving, the period covering pregnancy (conception to subsequent calving) using days from conception, and a data set combining these two periods. In the combined model, the two time scales, days from calving and days from conception were retained. The fitting of each set is described in turn.

For the lactation only data set, eight models were fitted, four models with a full covariance matrix for the random effects, and four models with the random effects assumed to be independent. The four models used were models including: full interaction between breed and parity, breed and parity but no interaction between them, breed only, and a model with no systematic effects. (Comparison of the two latter models showed that breed should be in the model, and thus we did not fit a model with only parity as systematic effect.)

Comparing AIC values (which should be as small as possible) it was found that the model with both breed and parity, but no interaction between them, described the data better than the other models (Table 2). With regard to the type of random effects, the AIC values were smallest for the models with independent random effects.
Table 2 The effect of different combinations of fixed factors for breed and parity, and different covariance structures for the random effects, on model fit. Values for model fit are given as Akaike's Information Criterion values for each of the three data periods: lactation only, pregnancy and the combined lactation and pregnancy data (fitted with and without an offset, $\partial R$ )

Fixed effects included in the model out of: breed (B), parity

\begin{tabular}{|c|c|c|c|c|}
\hline \multirow{2}{*}{$\begin{array}{l}\text { Covariance structure } \\
\text { assumed for the random } \\
\text { effects }^{\dagger}\end{array}$} & \multicolumn{4}{|c|}{$(\mathrm{P})$, and breed $\times$ parity $(\mathrm{B} \times \mathrm{P})$} \\
\hline & None & B & $B, P$ & $\mathrm{~B}, \mathrm{P}, \mathrm{B} \times \mathrm{P}$ \\
\hline \multicolumn{5}{|l|}{ Lactation only model } \\
\hline Independent & 17922 & 17847 & 17844 & 17849 \\
\hline Dependent & 18182 & 18181 & 18110 & 18124 \\
\hline$\rho_{\alpha \beta}($ rest $=0)$ & 17754 & 17710 & 17709 & 17718 \\
\hline$\rho_{\alpha \beta}, \rho_{\beta \lambda}$ & 17756 & 17712 & 17711 & 17720 \\
\hline$\rho_{\alpha \beta \prime} \rho_{\alpha \lambda}$ & 17746 & 17703 & 17703 & 17710 \\
\hline \multicolumn{5}{|l|}{ Pregnancy model } \\
\hline Independent & 15102 & 15036 & 14990 & 15008 \\
\hline Dependent & 15069 & 15008 & 14967 & 14986 \\
\hline$\rho_{\alpha \xi}($ rest $=0)$ & 15088 & 15015 & 14970 & 14986 \\
\hline$\rho_{\alpha \xi^{\prime}} \rho_{\xi \kappa}$ & 15087 & 15011 & 14965 & 14984 \\
\hline$\rho_{\alpha \xi}, \rho_{\alpha \kappa}$ & 15090 & 15014 & 14968 & 14986 \\
\hline \multicolumn{5}{|c|}{ Combined model without the parameter $\partial \mathrm{R}$} \\
\hline Independent & 21605 & 21541 & 21523 & 21517 \\
\hline Dependent & 21417 & 21369 & 21367 & 21365 \\
\hline \multicolumn{5}{|c|}{ Combined model with the parameter $\partial \mathrm{R}$} \\
\hline Independent & 21461 & 21371 & 21339 & 21364 \\
\hline Dependent & 21290 & 21241 & 21232 & 21253 \\
\hline$\rho_{\alpha \beta}, \rho_{\alpha \kappa}, \rho_{\beta \kappa}$ & 21339 & 21276 & 21242 & 21261 \\
\hline
\end{tabular}

${ }^{\dagger}$ Individual covariances between parameter pairs are referred to using the symbol $\rho$ with subscripts identifying the parameter pair according to the notation given in the Material and methods section. The structure 'Independent' assumes that all covariances $=0$, 'Dependent' indicates that all covariances were estimated. When individual covariances are specified, the other covariances in the model were set to 0 .

Adding a term to the model with independent random effects for the correlation between the random effect of $A$ and the random effect of $R\left(\rho_{\alpha \beta}\right)$ decreased the AIC values. Thus we considered the models with this correlation. Adding to this model a further term, for the correlation between the random effect of $R$ and the random effect of $I\left(\rho_{\beta \lambda}\right)$ increased the AIC values but adding a term for the correlation between the random effect of $A$ and the random effect of $I\left(\rho_{\alpha \lambda}\right)$ decreased the AIC values. Thus, for the final estimates of the effects of breed and parity on lactation curves of condition score, we selected a model that included $\rho_{\alpha \beta}$ and $\rho_{\alpha \lambda}$, i.e. the correlations between the random effect of $A$ and the random effect of $R$ and correlation between the random effect of $A$ and the random effect of $l$.

Normal probabilities plots (q-q-plots) and histograms of the residuals and random effects did not show deviance from normality. Random effects plotted pair-wise against each other confirmed the selected covariance structure.

A similar process of fitting eight models to the pregnancy data set was carried out. As with the pre-pregnancy data, it was found that the model with both breed and parity, but no interaction between those, described the 
data better than the other models (Table 2). With this data set, an interaction between the random effect of $A$ and the random effect of $S$ and an interaction between the random effect of $S$ and the random effect of $k$ were found.

For the combined pre and post pregnancy data set, the models were fitted either without the parameter $\partial R$ (forcing the levels at the two calvings determining a full lactation period to be equal), or with the parameter $\partial R$. Models with individual coefficients for the two exponential terms, that is, models with $\partial R$, had smaller AIC values, and we should thus select a model with these two coefficients. (These models with two exponential 'opposite' exponential terms proved very hard to fit. In particular, the model with parity as systematic effect and all the random effects correlated could not be fitted satisfactorily.) We found no interaction between breed and parity in the model with the independent random effects. Thus, we considered the model with a systematic effect of both breed and parity. In this model, the random effect of $A$ and the random effect of $I$ were independent, and the random effect of $S$ and the random effect of $k$ were independent i.e. not significantly different from zero. From the model without the two correlations we successively eliminated correlations while getting a better AIC value until we got an AIC value of 21242 for the model with three correlations, between $A$ and $R$, between $A$ and $k$, and between $R$ and $k$. Again, for this correlation structure of the random effects, the model with both breed and parity as systematic effect but without interaction between the two, had the best AIC value.

\section{Effects of breed and parity on condition score curves}

The estimates of the curve coefficients as affected by breed and parity are given in Table 3 . The corresponding values of condition score $(\times 10)$ at the start of lactation and the nadir, together with the time to occurrence of the nadir, are given in Table 4. As might be expected, when the fitted values for the lactation only and pregnancy only models were plotted together (assuming conception occurred on day 90 of lactation) there were discontinuities (Figure 2) reflecting differences in the estimates of coefficient A (Tables 2 and 3). When the combined lactation and pregnancy model was used, the resulting fitted curves fell between the values based on lactation only and pregnancy only at the time point of overlap (day 90) suggesting that the combined model successfully stabilised the discrepancies at this point. Further discussion of the effects of breed and parity on these curves thus refers to the combined model fits. The fitted lactation curves of condition score ( $\times 10)$ for all parities of each breed are shown in Figure 3.

The fitted condition score values for the Danish Red breed were always greater than those of the Danish Holstein and Jersey breeds, reflecting significant differences in the individual curve coefficients (Table 3). This difference in condition score was significant for the nadir but was only a tendency at the start of lactation (Table 4). Thus the Danish Holstein and Jersey showed a greater loss of body condition in early lactation than the Danish Red. Condition score curves for Danish Holstein and Jersey were similar in all three lactations. First lactation animals in all three breeds had the shallowest curves in body condition with significantly different minima from second lactation cows. There were no clear, or significant, differences between second and third lactation; in the Danish Red the nadir of the curve was lowest for second lactation, the opposite was the case for Jerseys, and for Danish Holstein the curves were very close together. As there were fewer animals in third lactation these curves were associated with much larger standard errors. There were no significant differences between breeds in the time point in lactation when the minimum condition score occurred. However, the nadir was significantly later, on average 10 days, for first lactation cows (Table 4, Figure 3).

Table 3 The coefficients for fixed effects (intercept, breed and parity) describing the curves of condition score relative to days from calving ( $t_{i j}$ ) and days from conception $\left(s_{i j}\right)$ fitted for lactation only, pregnancy only or the combined data (the coefficients for which fixed effects are given are shown in bold)

\begin{tabular}{|c|c|c|c|c|c|c|c|c|}
\hline $\begin{array}{l}\text { Lactation only model } \\
\text { Pregnancy only model }\end{array}$ & $\begin{array}{l}Y i j= \\
Y i j=\end{array}$ & $\exp \left(\mathbf{A}_{\mathbf{i}}\right)$ & + & $\exp \left(\mathbf{R}_{\mathbf{i}}\right)^{*}$ & $\exp \left(-\exp \left(\mathrm{I}_{\mathrm{i}}\right)^{*} t_{i j}\right)$ & $\exp \left(\mathbf{S}_{\mathrm{i}}\right)^{*}$ & $\exp \left(-\exp \left(\mathbf{k}_{\mathbf{i}}\right)^{*}\left(283-s_{i j}\right)\right)$ & $\exp \left(A_{i}\right)$ \\
\hline $\begin{array}{l}\text { Intercept }^{\dagger} \\
+ \text { Parity } 2 \\
+ \text { Parity } 3 \\
+ \text { Danish Red } \\
\text { + Danish Holstein }\end{array}$ & & $\begin{array}{l}+0.926^{\mathrm{c}} \\
-0.021 \\
-0.035 \\
+0.181^{\mathrm{c}} \\
+0.029\end{array}$ & & $\begin{array}{l}+0.103^{c} \\
-0.026 \\
+0.143 \\
-0.290^{c} \\
-0.013\end{array}$ & $\begin{array}{l}-2.945^{\mathrm{c}} \\
+0.073 \\
-0.394^{\mathrm{a}} \\
+0.070 \\
-0.005\end{array}$ & $\begin{array}{l}+0.030 \\
+0.252^{\mathrm{b}} \\
+0.166 \\
+0.536^{\mathrm{c}} \\
+0.330^{\mathrm{b}}\end{array}$ & $\begin{array}{l}-4.758^{c} \\
+0.417^{\mathrm{a}} \\
-0.461^{\mathrm{a}} \\
-1.378^{\mathrm{c}} \\
-0.650^{\mathrm{b}}\end{array}$ & $\begin{array}{l}+0.961^{\mathrm{c}} \\
-0.040 \\
-0.109 \\
-0.135 \\
-0.082\end{array}$ \\
\hline $\begin{array}{l}\text { Combined model } \\
\text { Intercept }^{\dagger} \\
+ \text { Parity } 2 \\
+ \text { Parity } 3 \\
\text { + Danish Red } \\
\text { + Danish Holstein }\end{array}$ & $Y i j=$ & $\begin{array}{l}\exp \left(A_{i}\right) \\
+0.705^{c} \\
+0.014 \\
-0.165 \\
-1.106^{a} \\
-0.683^{b}\end{array}$ & + & $\begin{array}{l}\exp \left(\mathbf{R}_{\mathbf{i}}\right) * \\
+0.151^{\mathrm{c}} \\
+0.050 \\
+0.165 \\
-0.167^{\mathrm{a}} \\
+0.112\end{array}$ & $\begin{array}{l}\exp \left(-\exp \left(\mathrm{I}_{\mathrm{i}}\right) * t_{i j}\right) \quad+ \\
-3.173^{\mathrm{c}} \\
+0.123 \\
+0.080 \\
-0.198 \\
-0.154\end{array}$ & $\begin{array}{l}\exp \left(R_{i}+\partial \mathbf{R}_{\mathbf{i}}\right) * \\
+0.221^{\mathrm{a}} \\
+0.095^{\mathrm{a}} \\
+0.031 \\
+0.823^{\mathrm{c}} \\
+0.617^{\mathrm{c}}\end{array}$ & $\begin{array}{l}\exp \left(-\exp \left(\mathbf{k}_{\mathbf{i}}\right) \star\left(283-s_{i j}\right)\right) \\
-5.823^{c} \\
+0.439^{c} \\
-0.180 \\
-1.083^{c} \\
-0.589^{b}\end{array}$ & \\
\hline
\end{tabular}

${ }^{\dagger}$ The intercept gives the coefficient values for parity 1 Jerseys.

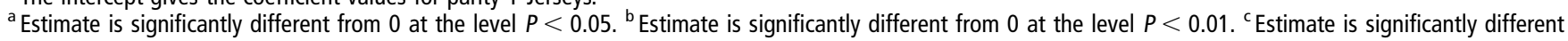
from 0 at the level $P<0.001$. 
Table 4 Fitted values of condition score at calving and the lowest point in curve, together with time of the lowest point after calving for the three breeds and parities. Estimates arising from the models for lactation only, pregnancy only and the combined data are given (where appropriate) (standard errors of the estimates are given in parentheses)

\begin{tabular}{|c|c|c|c|c|c|c|c|c|}
\hline & & \multicolumn{2}{|c|}{$\begin{array}{l}\text { Condition score } \\
\text { at calving }(\times 10)\end{array}$} & \multicolumn{3}{|c|}{$\begin{array}{c}\text { Condition } \\
\text { score at nadir }(\times 10)\end{array}$} & \multicolumn{2}{|c|}{ Time of nadir (days) } \\
\hline & & Lactation & Combined & Lactation & Pregnancy & Combined & Lactation $^{\dagger}$ & Combined \\
\hline Breed & Parity & & & & & & & \\
\hline \multirow{3}{*}{ Danish Red } & 1 & $3.9(0.09)$ & $3.9(0.08)$ & $3.0(0.08)$ & $2.3(0.23)$ & $3.2(0.07)$ & 53 (11.6) & $76(5.6)$ \\
\hline & 2 & $3.8(0.08)$ & $3.8(0.10)$ & $3.0(0.08)$ & $2.2(0.17)$ & $3.0(0.09)$ & $49(9.1)$ & $63(5.0)$ \\
\hline & 3 & $3.8(0.10)$ & $4.1(0.18)$ & $2.9(0.10)$ & $2.0(0.18)$ & $3.2(0.16)$ & $73(10.9)$ & $65(6.7)$ \\
\hline \multirow[t]{3}{*}{ Danish Holstein } & 1 & $3.7(0.07)$ & $3.8(0.08)$ & $2.6(0.06)$ & $2.4(0.18)$ & $2.8(0.07)$ & $57(8.6)$ & $79(5.5)$ \\
\hline & 2 & $3.6(0.07)$ & $3.6(0.09)$ & $2.5(0.07)$ & $2.3(0.13)$ & $2.5(0.09)$ & $53(8.0)$ & $68(4.6)$ \\
\hline & 3 & $3.7(0.10)$ & $3.9(0.17)$ & $2.5(0.09)$ & $2.1(0.16)$ & $2.6(0.14)$ & $79(13.9)$ & $69(8.0)$ \\
\hline \multirow[t]{3}{*}{ Jersey } & 1 & $3.6(0.08)$ & $3.7(0.08)$ & $2.5(0.06)$ & $2.6(0.12)$ & $2.7(0.08)$ & $57(8.1)$ & $79(5.3)$ \\
\hline & 2 & $3.6(0.08)$ & $3.6(0.09)$ & $2.5(0.07)$ & $2.5(0.09)$ & $2.5(0.09)$ & $53(8.0)$ & $73(6.3)$ \\
\hline & 3 & $3.6(0.10)$ & $3.6(0.13)$ & $2.4(0.08)$ & $2.3(0.15)$ & $2.3(0.13)$ & $79(14.1)$ & $71(8.2)$ \\
\hline
\end{tabular}

${ }^{\dagger}$ The time point at which condition score is $5 \%$ of the asymptote value $(\exp (A))$.

${ }^{\ddagger}$ Calculated assuming that conception occurred on day 90 of lactation.

Within the random effects, i.e. after adjustment for differences due to fixed effects of breed and parity, there was a significant negative correlation between the coefficient for the minimum value, $A$, and the increase above that value at calving, $R$. This was found for all three period models and was $-0.10,-0.084$, and -0.086 for lactation only, pregnancy only (correlation $S$ and $R$ ), and the combined data respectively. Although there were other significant correlations in the individual models (see above) there was no consistent pattern in them across models.

\section{Discussion}

The aim of this study was to model temporal patterns of condition score through lactation and pregnancy using a biologically interpretable function, and to derive estimates of the differences between breeds and parities. This has been achieved. The function used facilitates biological interpretation as its coefficients provide direct estimates of condition score at the start of lactation, end of pregnancy and the nadir of the condition score curve (Table 4). These quantities are important as it has previously been shown that changes in genetically driven body fatness can be predicted from them (Friggens et al., 2004). Genetically driven changes in body fatness affect prediction of intake (Friggens and Newbold, 2007) and have been shown to have a genetic correlation to traits such as fertility (Pryce et al., 2002).

There are other examples of the use of non-linear models with exponential functions in the agricultural domain, (e.g. Bermejo et al., 2003; Davidian and

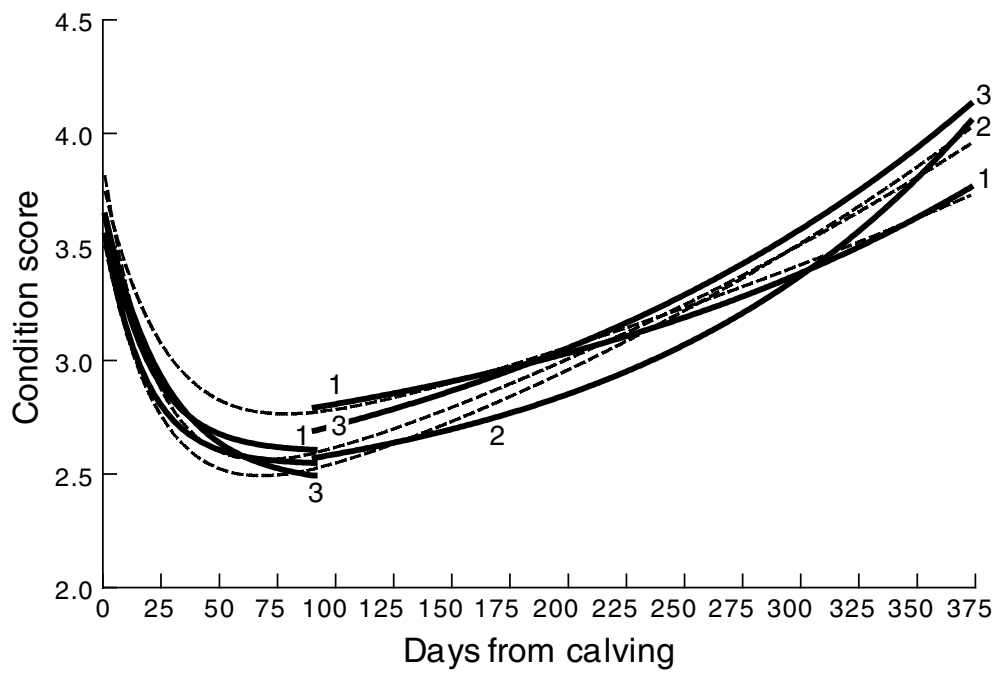

Figure 2 Example of the difference between the different time period models used for deriving curves in condition score for Danish Holstein cows in parities 1,2 , and 3 . The lactation only model is shown by the solid lines starting at 0 days from calving and truncated at 90 days from calving. The pregnancy model is shown as solid lines starting at day 90 , assumed to be day of conception, and continuing to the end of lactation. The combined model is shown by the stippled lines. Parity is indicated as 1, 2, or 3 for each curve. 

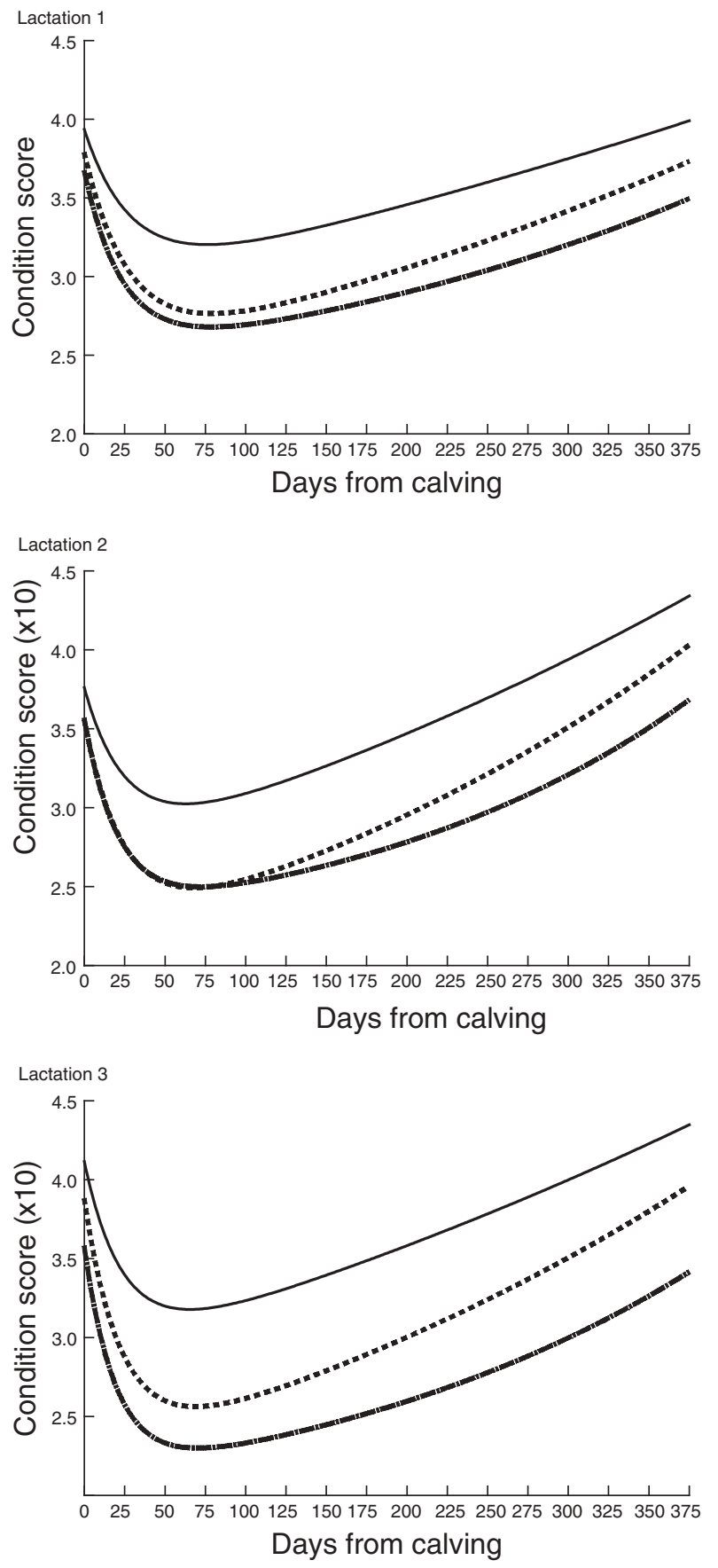

Figure 3 The effect of breed and parity on lactation curves of condition score estimated using the combined lactation and pregnancy model. For each lactation, breeds are Danish Red (solid line), Danish Holstein (even stippled line), and Jersey (uneven stippled line). Jersey had consistently the lowest condition score.

Giltinan, 2003 (section on Dairy science); Nielsen et al., 2004; Schinckel et al., 2005). In the present study, the intensity of recording was relatively limited (average eight observations per cow-lactation, range 1 to 17) and the measurement scale was relatively coarse $(0$ to 5 in $1 / 2$ units) compared with previous uses of such functions in agriculture. Thus the use of non-linear models to fit these data was not trivial. Indeed, simply using starting parameters derived by visual inspection of the data did not succeed. However, by careful selection of starting values we were successful in using the nonlinear mixed models on condition score data. This selection procedure used a stepwise method based on first fitting individual models to individual lactations to derive distributions of start parameters (see section Method for fitting models).

Significant and consistent differences in condition score curves of cows kept in the same environment under the same nutritional conditions were found. First lactation cows had shallower curves, they had greater condition scores at the nadir of the curve (Figure 3), which occurred approximately 10 days after the nadir of the curves for later lactations. This is in agreement with the findings of Dechow et al. (2002) and Gallo et al. (1996). The finding that younger animals invest less of their body reserves in lactation is in accordance with the observation that these animals generally have an additional energy demand for continued growth (Koenen et al., 1999). It also agrees with the accepted view in life history biology that investment in support of reproduction increases with age in mature mammals.

It should be noted that condition score is a subjective measure and thus direct comparison with other studies can be problematic. In the present study, the vast majority of scores were made by the same person who was periodically checked against external evaluators who used the condition scoring system on a wide range of farms. By this procedure we minimised variability due to differences between observers whilst maintaining the general scale for the Danish system (see Kristensen et al., 2006). However, there is a risk, inherent in this approach, that the single scorer begins to remember the scores of the cow from previous scorings and thus their judgement at any scoring is influenced by their prior knowledge. Although this type of bias cannot be ruled out it would, if marked, have been expected to produce notable discrepancies relative to the external evaluator. There was no evidence of this. A further issue relevant to the comparison of condition score curves between parities is that there fewer cows measured in third parity than in second parity, and likewise for parities 1 and 2. This was a feature of the experimental design with cows starting the experiment at first calving and some inevitably not completing three lactations. In consequence, the parameter estimates for third parity cows are less precise than those for second parity cows (Table 4). It is also possible that a bias was introduced in that not all cows were included in the estimates of the effects of the different parities. However, as the model used contained a random component to adjust for differences between cows, any such bias is likely to be negligible. Further, such a bias would only arise if there was a strong correlation between condition score curve shape and likelihood of being culled. We found no evidence that culled cows differed on average from other cows in their condition scores. 
Those breeds that have been subject to more intense selection for reproductive performance in the form of milk production, Danish Holstein and Jersey, were thinner at the end of the mobilisation period having lost more body condition than the Danish Red breed that has been selected for the dual purpose of both milk and meat production. Similar findings have been reported for both beef and dairy breeds of differing milk production potential (Sinclair et al., 1998; Dillon et al., 2003; Mao et al., 2004). There was also a tendency for Jerseys to have lower condition scores than Danish Holsteins but this difference was not significant. It is interesting to note that the breed differences in condition score curves were not obviously related to breed differences in size or milk production.

Given that these differences in the curves of body condition during lactation were found under stable and sufficient nutritional conditions, they imply that there is genetic variation in dairy cows innate drives to mobilise body reserves. If some mobilisation is genetically predetermined then it is reasonable to suppose that the cow is adapted to this type of mobilisation and that, therefore, it does not adversely affect health and reproduction. However, this assumes that genetically predetermined mobilisation which has arisen through natural selection, and is thus part of a successful reproductive strategy, should be benign otherwise it would not have been favoured. The extent to which this assumption is violated under artificial selection is ultimately a very important issue for dairy cow health and welfare, and for future sustainable genetic progress. Strong selection on only one component of fitness such as milk production may have result in a negative tradeoff with other fitness traits. Thus, if the trade-off gives rise to body mobilisation in excess of that which the cow is naturally adapted to we may find negative effects on health and reproduction (Friggens and Newbold, 2007). In this context, the ways in which the curves of body condition during lactation vary between individuals independent of nutritional treatments, and after adjustment for factors such as breed and parity, may provide insight into this process.

Because the non-linear models used here are more amenable to biological interpretation some further insights can be drawn from these results. Although the dairy breeds ended up being thinner there were no significant differences in the rate at which they lost body condition (exp $\left(l_{i}\right)$, combined model, Table 3). Within breed and parity, that is between animals of the same type, there was a substantial amount of variation in the nadir of the condition score curve (deviations in $\exp (A)$, Figure 4) i.e. some cows ended up thinner than others. However, there was no significant correlation between the level of condition at the nadir and the rate of condition score loss. Given that this result was found under long-term stable and sufficient nutritional conditions, it suggests that these are innate differences. Further, there was a consistent negative correlation between the level

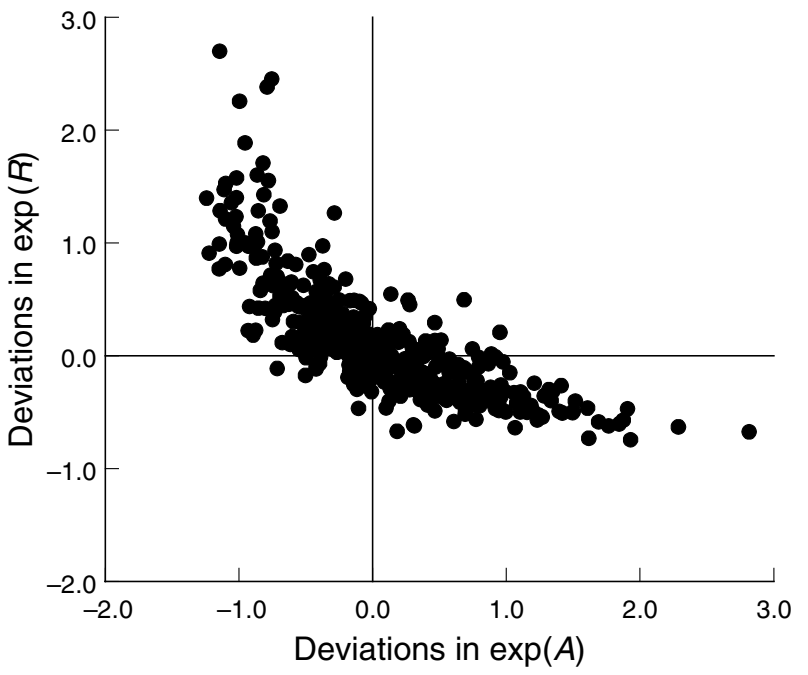

Figure 4 Individual cow deviations in the coefficients for the size of the decline in condition score $(\exp (R))$ and the nadir of the condition score curve $(\exp (A))$. Deviations are given in condition score units.

of condition at the nadir (deviations in $\exp (A)$ ) and the size of the difference between condition score at calving and at the nadir (deviations in $\exp (R)$ ). As shown in Figure 4 , the slope of this relationship was close to -1 . This indicates that the between cow variation in condition score at the nadir is not related to condition score at calving since differences in condition score at the nadir are largely compensated for by differences in the size of the drop. This supports the view (Ingvartsen et al., 1999; Friggens, 2003) that the frequently observed negative relationship between condition score level at calving and rate of condition score loss (e.g. Broster and Broster, 1998) is not a direct genetic effect but rather is primarily due to prior nutritional insult, i.e. it is a permanent environmental effect. Cows appear to vary in their innate propensity to lose condition independent of nutritional conditions and of condition score at calving.

\section{Conclusion}

Temporal patterns in condition score of cows kept under stable and sufficient nutritional conditions can be successfully described using a two component non-linear function. Further, there were significant differences between breeds and parities in the parameters of these curves.

\section{Acknowledgements}

We gratefully acknowledge the valuable contribution of Gitte Munch who did all the condition scoring, Hanne Marie Nielsen, Peter Trier, Connie Middelhede, and the farm staff at Ammitsbøl Skovgård. This study, which was financed by the Research Centre for the Management of Animal Production and Health (CEPROS), used data from the MEMO project funded by the Danish Ministry of Food, Agriculture and Fisheries and the Danish Cattle Industry via Finance Committee Cattle. 


\section{References}

Banos G, Coffey MP and Brotherstone S 2005. Modeling daily energy balance of dairy cows in the first three lactations. Journal of Dairy Science 88, 22262237.

Bermejo JL, Roehe R, Schulze V, Looft H and Kalm E 2003. Genetic change of feed intake curves in growing pigs using non-linear two-stage genetic analysis and linear random regression models. Journal of Animal Breeding and Genetics 120, 217-227.

Broster WH and Broster VJ 1998. Body score of dairy cows. Journal of Dairy Research 65, 155-173.

Burnham KP and Anderson DR 2002. Model selection and multimodel inference, second edition. Springer Verlag, New York.

Chilliard Y, Ferlay A, Faulconnier Y, Bonnet M, Rouel J and Bocquier F 2000. Adipose tissue metabolism and its role in adaptations to undernutrition in ruminants. Proceedings of the Nutrition Society 59, 127-134.

Coffey MP, Emmans GC and Brotherstone S 2001. Genetic evaluation of dairy bulls for energy balance traits using random regression. Animal Science 73, 29-40.

Correa MT, Curtis CR, Erb HN, Scarlett JM and Smith RD 1990. An ecological analysis of risk factors for postpartum disorders of Holstein-Friesian cows from thirty-two New York farms. Journal of Dairy Science 73, 1515-1524.

Davidian M and Giltinan DM 2003. Nonlinear models for repeated measurement data: An overview and update. Journal of Agricultural Biological and Environmental Statistics 8, 387-419.

Dechow CD, Rogers GW and Clay JS 2002. Heritability and correlations among body condition score loss, body condition score, production and reproductive performance. Journal of Dairy Science 85, 3062-3070.

Dijkstra J, France J, Dhanoa MS, Maas JA, Hanigan MD, Rook AJ and Beever DE 1997. A model to describe growth patterns of the mammary gland during pregnancy and lactation. Journal of Dairy Science 80, 2340-2354.

Dillon P, Buckley F, O'Connor P, Hegarty D and Rath M 2003. A comparison of different dairy cow breeds on a seasonal grass-based system of milk production 1. Milk production, live weight, body condition score and DM intake. Livestock Production Science 83, 21-33.

Friggens NC 2003. Body lipid reserves and the reproductive cycle: towards a better understanding. Livestock Production Science 83, 219-236.

Friggens NC and Newbold JR 2007. Towards a biological basis for predicting nutrient partitioning: the dairy cow as an example. Animal 1, 87-97.

Friggens NC, Ingvartsen KL and Emmans GC 2004. Prediction of body lipid change in pregnancy and lactation. Journal of Dairy Science 87, 988-1000.

Gallo L, Carnier P, Cassandro M, Mantovani R, Bailoni L, Contiero B and Bittante $G$ 1996. Change in body condition score of Holstein cows as affected by parity and mature equivalent milk yield. Journal of Dairy Science 79, 1009-1015.

Hansen JV, Friggens NC and Højsgaard S 2006. The influence of breed and parity on milk yield, and milk yield acceleration curves. Livestock Science 104, $53-62$.
Ingvartsen KL, Friggens NC and Faverdin P 1999. Food intake regulation in late pregnancy and early lactation. In Metabolic stress in dairy cows (ed. MG Diskin), British Society of Animal Science occasional publication no. 24. BSAS, Edinburgh, pp. 37-54.

Jorritsma R, Jorritsma $H$, Schukken $\mathrm{YH}$, Bartlett PC, Wensing T and Wentink $\mathrm{GH}$ 2001. Prevalence and indicators of post partum fatty infiltration of the liver in nine commercial dairy herds in The Netherlands. Livestock Production Science 68, 53-60.

Koenen EPC, Groen AF and Gengler N 1999. Phenotypic variation in live weight and live-weight changes of lactating Holstein-Friesian cows. Animal Science 68, 109-114.

Kristensen E, Dueholm L, Vink D, Andersen JE, Jakobsen EB, Illum-Nielsen S, Petersen FA and Enevoldsen C 2006. Within- and across-person uniformity of body condition scoring in Danish Holstein cattle. Journal of Dairy Science 89, 3721-3728.

Kristensen T 1986. Method for estimation of body condition of dairy cows. Report no. 615. National Institute of Animal Science, Denmark.

Littell RC, Milliken GA, Stroup WW, Wolfinger RD and Schabenberger 02006. SAS for mixed models. SAS Institute Inc. Cary, NC, USA.

Lowman BG, Scott N and Somerville S 1976. Condition scoring of cattle. Technical bulletin no. 6. East of Scotland College of Agriculture, Edinburgh, Scotland, pp. 1-29.

Mao IL, Sloniewski K, Madsen P and Jensen J 2004. Changes in body condition score and in its genetic variation during lactation. Livestock Production Science 89, 55-65.

Nielsen HM, Friggens NC, Løvendahl PL, Jensen J and Ingvartsen KL 2003. Influence of breed, parity and stage of lactation on lactational performance and the relationship between body fatness and live weight. Livestock Production Science 79, 119-133.

Nielsen OK, Ritz C and Streibig JC 2004. Nonlinear mixed-model regression to analyze herbicide dose-response relationships. Weed Technology 18, 30-37.

Petruzzi H, Danfær A and Friggens NC 2004. A dynamic model of feed intake regulation in dairy cows. Model evaluation. Journal of Animal and Feed Sciences 13, 25-49.

Pryce JE, Coffey MP, Brotherstone S and Woolliams JA 2002. Genetic relationships between calving interval and body condition score conditional on milk yield. Journal of Dairy Science 85, 1590-1595.

Schinckel AP, Adeola 0 and Einstein ME 2005. Evaluation of alternative nonlinear mixed effects models of duck growth. Poultry Science 84, 256-264.

Sinclair KD, Yildiz S, Quintans G and Broadbent PJ 1998. Annual energy intake and the performance of beef cows differing in body size and milk potential. Animal Science 66, 643-655.

Wolfinger RD 1999. Fitting nonlinear mixed models with the new NLMIXED procedure. Proceedings of the SAS SUGI conference, Miami Beach, Florida, pp. 287. 